cembre 188r, portant nutamment protongation de la duréc-de la concession, la Sociéte Lyonmaise prend l'engagement de faire l'écharage électrique du bonlevart de la Gare pendant to mois aux conditions et prix convemus of que, moycmnant cet essai, il est entendu que, si la commme d'llyeres se décide à un moment quelconque à adopter l'éclairage électrique, elle domera à la Société Lyomnase la préférence à tous antres demandeurs de la concession exclusive de ce mode d'éclairage à conditions égales ;

III. Considérant que l'article 13 précilé du traité de 1867 est au nombre de ceux que l'article ro du traité de r88r déclare maintenues en ce qu'ils nont rien de contraire aux nouvelles conventions; que du rapprochement des deux traités, il résulte que la ville d'flyères, an moment où elle accordail à la Société une prolongation de concession, a entendu, lout en maintenant les droits résullant pour elle de l'article r3 du traté de 1867 , s'assurer la faculté, en dehors du cas prévu par cet article, d'adopter l'éclairage électrique, sons la seule réserve du droit de préférence à conditions egales pour son concessionnaire du gaz;

IV. Hais considerant que d'apres la commune intention des partics, la preférence is condilions egales suppose nocessairement des propositions offrant des garantics sérieuses et portant sur des chauses pratiguenent exombables ef permellant une exploitation normale et rémmótutrice; que l'étal de l'instrution ne permet pas de recomantre si lo tailé proposé par le sicur Gauthier répond aux condilions indiquées ci-dessus; qu'il y a done lieu, avant dire droil, d'ordonner une expertise; Décide:

Arr. $1^{\text {er }}$. 11 sera, avant dire droit au fond, procédé à une expertise à l'effel de déterminer si le traité offert à la ville par te sieur Gauthier el Gie offre des garunties séricuses, si les clauses sont pratifuement exćcutables et pernellent une exploitation normale el rómunératrice ;

Fante par les parties de s'entendre sur le choix d'un expert unicue, la ville dllyeres el la Société lyomaise nommeront chaaune un expert el le troisiene expert sera désigné par le président de ha Section du Contentieux du Conscil d'Elat; le ou les experts prêteronl sement entre les mains du vice-président du Conseil de prefexhe du Var. Ils déposeront leur rapport au Secretarial du Contenteux du Conseil d'blat dans un délai de 3 mois à partir de la prestation de serment;

Art. 2. - Les dépens sont résurvés; il y sera statué en fin l'instance.

Paul Bozgault, Avocal à la Cour d'Appel de Lyon.

\section{LES TORRENTS DE LA SAVOIE}

M. P. Movan, Mnspereur des Hanx of Forets, vient de publier un volume grand in-80 de 1 sho pages sur les torrents de la Savole. Ciest la dresciplion do tous les coms d'aul de celte région des Alpes el lhistoriciue des travaux. molrepris pour la conservation du régime des eaux, la corpretion du lit des forrents, la defense contro les inondations, rle... La docmontalion est formidable el d'me valeur sans cogale. Ce travail fail le plus grand homneur à M. Mouon, dont nous avons dejia en somvent l'oecasion de citer les travaux dans celle Rerme.

Nous mellons sous les youx de nos lecteurs quolques-unes des pages de ce rolume qui pamronl le mienx leur donner uno idée de lintérel gưil présmte.

\section{L'ORIGINE DES TORRENTS EN SAVOIE}

DESCRIPION TOPORAAPIIQTE SOMMAIRE DE IA SIVOHE

L'ancien duché de Savoive, rémi à la Fiance depuis r860, forme les deux départements de la Savoie et de la llauleSavoie; il a une superficio de ro of 6 kilomètres carrés. II csl compris entre 45 degrés o5’ el 46 degrés 25 de lalilude Nord, 3 degrés I 7' et 4 degrés 5 r' de longitude Est.

Du còlé de l'Est, cette région confine au royaume d'Ilaiic el à la République llelvélique dont la séparent la chaîne principale et un contrefort des Alpes; au Nord, le Lac Léman, puis une ligne conventionnelle quiltant le lac près dllermance et aboulissant à Chancy sur le Rhòne constiluent la fronlière franco-suisse. Partout ailleurs, la Savoic touche au lerritoire français. A l'Ouest, sa limite suit le Rhòne puis son aflluent le Guiers; au Sud, clle est forméc par les Alpes de Maurienne'se délachant au Thabor de la chaîne frontière, puis par le Bens, affluent du Bréda, qui lui-même se jelle dans l'Isc̀rc, et enfin par une ligne conventionnelle altcignanl les falaises qui terminent le massif de la Charteuse.

Les allitudes extrêmes sont 2 I 2 mètres au confluent du Rhône et du Guiers el 48 ro au sommet du Mont-Blanc. Entro cos deux points, la distance n'est que de roo kilomètres à vol d'oiseau.

En quittant les bords du Rhòne et en s'avançant vers l'lalic, on rencontre d'abord des monlagnes peu élevécs, calcaires ou gréseuses : ce sont les Préalpes et le Jura. Les Voirons $\left(\mathrm{r} 486^{\mathrm{m}}\right)$, le Salève $\left(\mathrm{r} 38 \mathrm{o}^{\mathrm{m}}\right.$ ), le Vuache (I I I I $\left.{ }^{\mathrm{m}}\right)$, la chaîne de la Chambolle $\left(852^{\mathrm{m}}\right)$, et celle de l'Epine $\left(1497^{\mathrm{m}}\right)$ lcur appartienment et sont comme les ouvrages avancés, échelonnés tout près du fleuve, qui défendent l'accès du grand massif alpin.

A bonne distance en arrière el séparés par les larges glacis du lac du Bourget, du bas Chéran et de la Fillière, se dressent de hauts remparts calcaires découpés par de profondes cluses. Des vallées ramifiées, souvent étranglées par les rocs, ouvertes vers le Nord-Ouest ont permis d'aborder et de parcomrir tous les replis de ces chaînes dites subalpines. Du cốté de l'Orient, ces divers groupes de montagnes se terminent par des falaises ou des escarpements que, seuls, de rares passages interrompent. Lcur autonomie s'accuse également dans les noms particuliers qu'jls ont reçus.

C'est d'abord le massif des Dranses formant l'arrondisscmont de Thonon ct une très faible partic de celui de Bonneville, au Sud du lac Léman, séparé cn deux parties pár la Dransc, depuis la Tête de Bostan $\left(2.4 \mathrm{II}^{\mathrm{m}}\right)$, à la frontièrt suisse, jusqu'à Bioge el ensuite, de là à son embouchure, dans le lac suivant une dépression orienlée S.S.E.-N.N.W.

La région de l'Esl est parcourue par la Dranse d'Aboildance cl celle de l'Ouest par la Dranse de Belleveaux ou Brevon qui se réunissent toutes deux près de Bioge, à ro kijlomètres en amont de Thonon à la Dranse de Morzine. L'cnsemble de ces vallées et les bords du Léman constituent te Chablais. Au Sud du massif et orienté sensiblement E.-W. coule le Giffre descendu des glaciers du Mont-Ruan (3 o $7^{6^{\mathrm{m}}}$ ) également à la frontièrc suisse; il passe à Sixt, à Samoens el à Taninges et se jette dans l'Arve à Marignier.

Sur la rive gauche de cette dernière rivière, artère du Faucigny, se dressent les escarpements du massif des Bornes, compris dans le quadrilatère: Sallanche, Bonneville, Annecy, U'gines. Le principal cours d'eau qui en sort eșt lo Fier qui, né au Mont-Charvin (2/4 $\left.4^{m}\right)$, coule du S.-S.-E au N.-N.-W. jusqu’à la gorge de Saint-Glair et reçoit, à droile, a Thones, le torrent du Nom, venu du col des Aravis ( 1498 ). Ce col, un des plus beaux de la région, met en communication la valléc du Fier avec celle de l'Arly. Toute la portion septentrionale de celte région verse ses eaux dans le Borñe venu de Pointe-Percée $\left(27^{5} 2^{\mathrm{m}}\right)$ et qui a donnẻ son nom all massif. 
Au Sud ef au S.-W. des Bornes el les séparant des Beanges se creusont les vallées du lac d'Annecy, de l'Fan-Morte, el te la Chaise se réunissant au col de Faverges, dont l'allitude n'est que de 5oo mètres.

C'est le Chéran venant de la pointe Chaurionde $\left(2297^{\mathrm{m}}\right)$ et dont la direction généraJe est encore du S.-F. au N.-W., qui sepl d'émissaire aux eaux de toules les montagnes des Boauges ol les amène au Fier près de Rumilly.

I. bassin de Chambéry, qui n'est autre que celui du lac du Bourget at de son aflluent, le torrent de Teysse, descendu des penles S.-W. des Bauges, fait communiquer le Rhône ave l'Jsère par le seuil peu élevé de Chignin $\left(30{ }^{\mathrm{m}}\right)$. Dominé it l'Est par les Beauges, il l'est au S.-W. par les cimes septentrionales de la Chartreuse, dont le point culminant dans la région est le. Mont-Granier ( $\mathrm{g} 3 \mathrm{~S}^{\mathrm{m}}$ ), à la limite de la Savoie ol du Dauphiné.

Toules ces cluses transversales qui enlaillent si profondénent los falaises calcaires semblent être des canaux d'écoulrment ménagés par la nature aux caux de fusion des glacicrs des hautes régions siluces plus à l'Fst. Et, en effel, on trouve sur les lerrasses qui bordent ces vallées la preuve indisculable qu'autrefois l'Isère et-le Doron de Beaufort gagnaienl directement le Rhòne, l'une par le lac du Bourget "l'aule par lo lac d'Annecy, comme aujourd'hui cncore l'Arve par le défilé de Magland.

Il a fallu des phénomèncs de capture pour donner au Doron of à l'lsère leur direction achelle au travers de Ja large (1) superbe vallée que l'on nomme d'abord le Grésivaudan, puis Ja Combe de Savoic. La vallée de l'Isère continuée par eclle de l'Arly, le col largement échancré de Mégève, la vallée de l'Arve après celle de la Diosaz, le col d'Anterne rt lo Ilaut-Giffre, telle est la ligne de démarcation entre les chaincs subalpines et les Alpes proprement dites.

D'une façon générale, les Alpes occupent en Savoie une surface Iriangulaire affectanl la forme d'un sccleur circulaire dont te sommet serail au Mont-Blanc (/ $8 \mathrm{ro}^{\mathrm{m}}$ ), dont les lignes joignant celte sommité à la Levanna $\left(36 \mathrm{I} 9^{\mathrm{m}}\right)$ en suivant la fronlière franco-italienne d'une part, au Puy-Gris (2. $g{ }^{\mathrm{m}}{ }^{\mathrm{m}}$ ) à la limite du département de I'Isère, d'autre part, seraient les rayons cxtrêmes.

Entre la chaìne frontière el celle qui, parallèlement à la dípression de l'Isère, relie le Monl-Blanc à Bclledonne s'insère une sćrie d'aulres chaînons disposés en éventail et orientés entre N.-S. et N.E.-S.W. D'importantes vallées, presque parallèles, concentriques, incurvées vers le. Sud, coupent sucessivement ces diver's ressauts el atlcignent jusqu'aux extrémilés mêmes du territoire.

Au Ford, on trouve d'abord l'Arve, sortie dı col de Balme (2 $202^{\mathrm{m}}$ ), qui longe la chaîne du Mont-Blanc, rasscmble les caux do nombreux lorrents glaciaires parmi lesquels il faul ciler l'Arveyron et le nant de la Griaz; elle lourne ensuite à l'Ouest à partir des Houches, reçit à droile la Diosaz descondue du Buct $\left(3 \log ^{\mathrm{m}}\right)$, le nant Noir sorti des éboulis du Dérochoir ; à gauche, lc nant Nalien; venu du col de Voza $\left(\mathrm{r} 6-7^{\mathrm{m}}\right)$ at, au Fayel, le. Bonnant, issu du col de Bonhomme $\left(23.40^{\mathrm{m}}\right)$, qui parcourt le val de Montjoie en recueillant les torrents des glaciers du Sud du Mont-Blanc, ainsi que lo nant Borrant el le nant Rouge, plaies vives ouvertes dans lo flanc oriental du Mont-Joly. A partir de Domancy, l'Arve guilte la zone alpine ef remonte vers le Nord.

A 40 kilomètres plus au Midi, s'ouvre une longue vallée, la plus importante de la Savoie, celle de l'Tsère qui, depuis son origine au glacier de la Galisc jusqu'à Bourg-Saint-
Maurice, court en remontant vers le Ford parallèlement à lat frontière franco-italienne.

De Bourg-Saint-Maurice $\left(815^{-m}\right)$ à Albertville $\left(3 / 40^{m}\right)$, l'Isere s'infléchil d'abord vers le S.-W. jusquà Mouliers (hos? d'où elle rebrousse brusquement vers le $\mathrm{N}$. W. pour renir déboucher sous la lerrasse de Conflans, à l'origine de la Combe de Savoic. De nombreux cours d'eau viennent dans lout ce long trajel se jeter dans l'Tsère. Parmi les plus importants on peul citer : ä droite, le Versoyen grossi du lorrent des Glaciers, dont le bassin, par le col de la Scigne. $\left(25 \mathrm{r}^{\mathrm{m}}\right)$ et le col du Bonhomme $\left(2340^{\mathrm{m}}\right)$ communique ave le Val Veni et la vallée de Montjoie.

A gauche, à Moulicrs, l'Isère est presque doubléc par le. Doron. Le Doron, appelé aussi Doron de Bozel on de Pralognan, a un bassin très considérable couronné au sud ri a l'Est par les imporlantes nappes glaciaires de Polsel of d. la Vanoise; il est grossi à droite par le Doron de Champagny, à gauche par le Doron de Belleville accru du ruisseau des Encombres dont J'origine est au col de mêmo nom $\left(2 / 2 \mathrm{r}^{\mathrm{m}}\right)$ qui mène dans la vallée de l'Arc.

Tout le bassin de l'lsère el de ses affluents compris dans la région alpine a reçu le nom de Tarentaise. La Tarentaiso englobail aussi loul le bassin du Doron de Beaufort. Celle rivière, affuent de gauche de J'Arly, qui lui-même rejoint l'Tsère sous Alberlville, a une dircclion générale E.-W. Elle nâ̂t près du col de Roselend qui conduit dans la vallée des Glaciers, reçoit à gauche lo nant de Treicol, puis, à Beaufort, l'Argentine ou ruisseau d'Arèches, dont la valléc correspond arec colle de l'Ormente par le Cormet d'Arèches ( $\left.194^{\prime m} /^{m}\right)$ cl avec celle du nant Bénétant par le col de la Bathie ( $\left.1906^{\mathrm{m}}\right)$.

De même que les bassins des Dranses, de l'Arve ef de l'Isère, celui de l'Arc a regu un nom spécial : il forme à lui seul la Maurienne.

Mais à la différence de l'Isère et de l'Arve, loul le cours de l'Arc se trouve dans la zone alpine. I'Arc sort du glacier des sources de l'Arc, à 28 ro mètres, an pied de la Jevanna $\left(36 \mathrm{I} 9^{\mathrm{m}}\right)$; jl doit son nom à la coumbe qu'il décrit, dont la concavilé est dirigée du côlé du Nord el dont le point le plus méridional est au village des Fourneaux. Il est, à son origine, puissamment alimenté par les importants glaciers te platraux qui marquent la frontière franco-italienne, jusqu'à Bessans $\left(\mathrm{r} 799^{\mathrm{m}}\right)$. En aval de celte localilé, il reçoil à droile : Ic Doron de Termignon ( $12.80^{\mathrm{m}}$ ) qui se forme à Entre-deux-Eaux, au pied du col de la Vanoise $\left(2 / 173^{m}\right)$ des deux torrents la Jeysse et la Rocheure, émissaires des produils de la fusion d'un cirque de petils glaciers.

En aval de Modane, le Poussel, Ja Grollaz, Ie torrent de Saint-Martin, le Rieu Sec, to Cilarel et to Saint-Julien sont les affluents de droile les plus importants. On trouve onsuite, en descendant la vallée, les ruisscaux d'IJermillon, et de Pontamafrey, te Bugeon qui prend sa source au col de la Madeleine ( $984^{\mathrm{m}}$ ), les ravins de la Chapelle, des Moulins d'Fpierre, de la Balme et de Montarticr, le ruisseau de la Roche venant du col de Basmont ( $600^{\mathrm{m}}$ ) el cnfin le Vorgeray qui longe le versant oceidental du Grand Arc $\left(2489^{\mathrm{m}}\right)$.

A gauche, l'Arc recoil anssi, depuis Lanslebourg, un nombre considérable de tributaires, parmi lesquels il faut mentionner les torrents de l'Envers, du nant el de SaintAntoine, le Charmaix, la Neuvache el la Valloirette qui descend du massif du Galibier, le Rieu-Bel à Villargondran et, à St-Jean-de-Maurienne $\left(533^{\mathrm{m}}\right)$, Io boueux Arvan, grossi du Merderel deVillarembert, du Merderel des Albiez et du Bon-Rieu de Jarrier ; püs, à Saint-Etienne-de-Cuines $\left(/ 155^{\mathrm{m}}\right)$, 
le Glandon venu du col de ce nom ( $195 \mathrm{I}^{\mathrm{m}}$ ), accru des eaux du ravin de Niolan, enfin le torrent de la Corbière à SaintPierre-de-Belleville et le ruisscau de la Croix-d'Aiguebelle

De ce que les trois grandes vallées de l'Arve, de l'Isère el de l'Are, coupent plus ou moins obliquement les chaines alpines résulte celle conséquence que les lignes de faîte qui les séparent ont un relief très irrégulier; elles atteignent une altilude considérable, lorsqu'elles arrivent au sommet d'un des plissements pour relomber à un niveau souvent assez bas, immédialement en avant ou en arrière.

Entre le bassin de l'Arve el celui de l'Isère, l'arête de parlage des eaux part de l'Aiguille des Glaciers $\left(3634^{m}\right)$, au Sud du massif du Mont-Blanc, gagne la pointe des Fours $\left(276 \mathbf{r}^{\mathrm{m}}\right)$, le col du Bonhomme (2 $\left.3 / \mathrm{ho}^{\mathrm{m}}\right)$, la pointe de Rosellette $\left(269 \mathrm{o}^{\mathrm{m}}\right)$, le col du Joly ( $\left.999^{\mathrm{m}}\right)$, les $\Lambda$ iguilles du Joly $\left(2487^{\mathrm{m}}\right)$ et le col de Mégève ( $12 \mathrm{I}^{\mathrm{m}}$ ) qui sert de démarcation entre les zones alpine el subalpine.

La crêle qui court cnlre le Doron de Bcaufort et l'Isère se soude à la précédenle à la pointe des Fours, descend au Cormet de Roselend $\left(\mathrm{r} 922^{\mathrm{m}}\right)$, remontè à la Grande Parei $\left(27^{3} \mathrm{I}^{\mathrm{m}}\right)$ ct conlinue à osciller, en passant par le Cormet d'Arèches ( $\left.\mathrm{x} 9 / 4^{\mathrm{m}}\right)$, Ie Cret du Rey $\left(268 \mathrm{~g}^{\mathrm{m}}\right)$, le col de la Louze (2 $\left.\times 25^{\mathrm{m}}\right)$, le Grand-Mont $\left(2698^{\mathrm{m}}\right)$, le col de la Bathie $\left(\mathrm{r}, 9 \circ 6^{\mathrm{m}}\right)$ el le Mirantin $\left(2,465^{\mathrm{m}}\right)$

Si l'on examine le massif monlagneux entre la Tarentaise el la Maurienne, on voit que les deux versants se coupent suivant une ligne briséc qui se détache à la cime d'Oin $\left(35 \mathrm{r} 4^{\mathrm{m}}\right)$ de la frontièrc italienne et alteint successivement le col du Mont-Iscran (2 $769^{\mathrm{m}}$ ), la pointe de Méan Martin $\left(3337^{\mathrm{m}}\right)$, la pointe de la Sana $\left(3450^{\mathrm{m}}\right)$, le col de la Leysse (2) $\left.78 \mathrm{o}^{\mathrm{m}}\right)$, l'Aiguille de la Grande-Molte $\left(3663^{\mathrm{m}}\right)$, le col de la Vanoise $\left(2 / 73^{\mathrm{m}}\right)$, la Dent-Parrachée $\left(37 \mathrm{r}^{\mathrm{m}}\right)$, le col de Chavière $\left(2806^{\mathrm{m}}\right)$, l'Aiguille de Polset $\left(3558^{\mathrm{m}}\right)$, le MontBroquin $\left(3 \times 94^{\mathrm{m}}\right)$, le col des Encombres $\left(2337^{\mathrm{m}}\right)$, le Grand Perron des Encombres $\left(2828^{\mathrm{m}}\right)$, le Grand Coin $\left(27 \mathrm{r} 7^{\mathrm{m}}\right)$, le Cheval Noir $\left(2834^{\mathrm{m}}\right)$, le col de la Madeleine (i $\left.984^{\mathrm{m}}\right)$, le Gros Villan $\left(2688^{\mathrm{m}}\right)$, le Bellachat $\left(2488^{\mathrm{m}}\right)$, le col de Basmont (r $\left.807^{\mathrm{m}}\right)$ et le Grand Arc $\left(2489^{\mathrm{m}}\right)$.

Du côlé du Sud, la Maurienne se termine par une nouvelle série de montagnes qui la sépare du Dauphiné. Cette limite part de la ligne frontière d'Italie au pic du Thabor $\left(3205^{\mathrm{m}}\right)$ pour arriver à la cime de Montgilbert (1 $47^{\mathrm{m}}$ ) par la roche du Grand Galibier $\left(3242^{\mathrm{m}}\right)$, le col du Galibier $\left(2658^{\mathrm{m}}\right)$, le pic des Trois-Evêchés $\left(3 \mathrm{I}_{2} \mathrm{~m}^{\mathrm{m}}\right)$, le pic de l'Etendard $\left(3473^{m}\right)$, le col de la Croix de Fer $\left(2088^{m}\right)$, la pointe de l'Ouillon (2 $\left.436^{\mathrm{m}}\right)$, le col du Glandon ( $\left.9^{5} \mathrm{r}^{\mathrm{m}}\right)$, les rochers d'Argentiers $\left(29^{1} 7^{\mathrm{m}}\right)$, le col du Merlet $\left(2294^{\mathrm{m}}\right)$, le pic du Frène $\left(2808^{\mathrm{m}}\right)$, la pointe du Rognier $\left(2346^{\mathrm{m}}\right)$ et le col du Grand Gucheron ( $1202^{\mathrm{m}}$ ).

Si l'on observe que la zone subalpine présente de nombreuses falaiscs calcaires dominant les pentes inférieures, que dans la région alpine des Aiguilles, des pointes rocheuses s'élancent fréquemment à plus de 3 ooo mètres, on peut en conclure que le ruisscllement doit s'exercer avec intensité el qu'outre les agents naturels de désagrégation, les actions érosives énergiques contribuent puissamment à modifier sans cesse le relief du sol. Comme il n'est pas rare de trouver dans les montagnes savoisiennes des versants dont l'inclinaison alteint et dépasse soo p. roo, il se produira donc des phénomènes torrentiels chaque fois que les conditions nécessaires pour leur donner naissance sc trouveront réunies.
DES GaUses de LA TORRENTIALITÉ. - DÉFinitions.

Dans le langage courant, on désigne par torrent tout cours d'cau descendant des ntontagnes. Combien de poètes ont chanté le murmure ou la plainte du ruisselet limpide I Qui n'a entendu célébrer les heurts, les violences contre les rochers de sombres gorges d'une eau rugissante, écumeuse qui semble vouloir ébranler les falaises d'alentour et se précipite par de presligieuses cascades dans les plaines qu'ellc anime et féconde ! Les torrents dont il sera ici question n'ont rien du charme qui a inspiré tant de gracieuses idylles ou de romantiques descriptions : ce sont des cours d'eau à crues subiles et viólentes, à pentes fortes et irrégulières. Ils affouillent, le plus ordinairement dans la montagne, déposent dans la pláine leurs matériaux de charriage en cxhaussant leur lit, ce qui a pour conséquence le déversemenl des filels liquides au moment des crues.

D'ordinaire, un torrent comprend trois parties bien distincles :

$I^{\circ}$ Le bassin de réception où se rasscmblent les eaux qui constituent le torrent cl où elles arrachent une grande partie des malérianx de charriage;

$2^{\circ}$ Le canal d'écoulement par où descendent vers la vallée principale les eaux venucs des régions supérieures;

$3^{\circ}$ Le cône ou lit de déjections, à l'issue du canal d'écoulement sur la plaine el où se forment des dépôts. Les mátières transportées proviennent ou bien du décapage par les agents atmosphériques (pluie, grêle, neige) de surfaces dénudées, ou bien des érosions des eaux dans les thalwegs, ou bien encore de glissements de berges ou de versants entiers, causés par affouillements ou excès d'humidité.

Depuis le milie'u du $\mathbf{x I x}^{\mathrm{e}}$ siècle surtout, de nombreuses personnes ont recherché les causes de ces érosions et de ces affouillements : parmi ces observateurs, il faut citer Alexandre Sunelt, ingénieur des Ponts et Chaussées, qui, en r845, a publié unc magistrale Etude sur les torrents des HautesAlpes, qui fail cncorc autorilé, et P. Demontzey, inspecteur général des Forêts, dont l'Etude sur les travaux de reboise: ment et de gazonnement des Montagnes a été le guide des forestiers des Alpes.

Combien d'autres administrateurs, ingénieurs ou foreștiers se sont occupés, tant en France qu'à l'Etranger, des graves problèmes que soulève la lutte contre les torrents !

Il résulte de l'expérience de près d'un siècle que les causès de la formation des torrents sont au nombre de trois qui sont plus loin étudiées en détail :

\section{$1^{\circ}$ La nature géologique du sol.}

Tout le monde sail que les divers terrains sont inégalement résistants. Alors que des roches cristallines ou sédimentaires sont très compacles, il se trouve des couches argileuses, gypseuses ou schisteuses très tendres, s'effritant à l'air, facilement attaquables par la gelée, le soleil et l'humidité. Il est clair qu'un courant d'eau, même très faible, aura vite fait de raviner un sol nu, formé des produits de désagrégation de ces dernièrés roches, landis qu'il arriveri à peine à entamer, au bout d'un temps très long, les affleurements granitiques ou calcaires sur lesquels il s'écoule. De même, les matériaux meubles, de faibles dimensions, cailloutis, alluvions, sables, dépôts fluviaux ou glaciaires se trouveront entraînés par le ruissellement, si rien ne vient réduire la vitesse el la force de propulsion des filets liquides.

$2^{\circ}$ Le climat.

G'est un fait d'expérience que les désastres causés par lé torrents ne se produisent qu'à la fonte des neiges ou ala 
suite de pluies d'orage. Dans la Savoice, où les monlagnes atteignent des altitudes considérables, il n'est pas rare de voir les pentes couvertes de neige forl tard se dépouiller en quelques jours de leur manteau hivernal. Souvent cn une scmaine, soleil et vent du Sud délịvrent les eaux immobiles, font verdir les pelouses el s'épanouir les fleurs des Apes. C'est encore le soleil qui dans les longs jours d'été aspirc l'humidité du sol que condensent en nuages et pluies les cimes élevées, les arêtes rocheuses et les aiguilles. L'orage alors déverse ses flots qui, suivant l'état du sol, s'écoulent lentement, inoffensifs, uliles même à la pérennité des sources ou se précipitent en mascaret terrible qui ravage tout sur son passage; seule, en effet, la végélation ligneuse peut ralentir sur les pentes le ruissellement et en supprimer les fàcheuses conséquences.

$3^{\circ}$ Le déboisement et les abus de jouissance.

Il n'est personne aujourd'hui pour mellre en doute les effets de la forêl que Surell a résumés dans les quatre propositions suivantes devenues de véritables axiomes:

a) La présence d'une forêt sur un sol empêche la formalion des torrents ; b) La destruction d'une forêt livre le sol en proie aux lorrents ; c) Le développement des forêts proroque l'extinction des torrents. ; d) La chute des forêts revivific les lorrents éteints.

\section{DE LA FORÊT, DE SON ACTION}

Depuis l'époque où le grand ingénicur condensait ainsi en formules le résullal de nombreuses observations, dans plusicurs pays d'Europe el notamment en France, en Allcmagne, en Autriche el en hussie, des savants, des forestiers, des agronomes éludiaient minutieusement l'influence sur los caux superlicielles ou souterraines des massifs boisés dont ils expliquaient ainsi scientifiquement l'action protectrice el bienfaisante.

Le fenillage persistant des essences résineuses reticnl du cinquième au ticrs de la lame d'cau qui tombe annuellement. Si la pluie est de peu de durée, il peut fort bien alriver qu'aucune partie n'cn arrive au sol. Toule cetle eau, interceplée par le couvert des arbres qui a aussi l'avantage de protéger le sol contre le choc mécanique des gouttes d'eau ou de la grêle, est rendıe à l'almosphère par évaporalion.

Dans les forêts feuillues de lorraine, la proportion de pluic retenue par les cimes ne scrait environ que le neuvième de la précipitation tolale.

M. le professeur IExry, de l'Ecole forestière de Nancy, estime loutefois qu'elle pourrait alleindre les trois dixièmes de cette précipitation, tandis que dans les futaies résineuses elle monterait jusqu’à la moitié.

De la pluic qui, au travers des branches el des feuilles ou des aiguilles, parvient jusqu'au terrain, une nouvelle quantité relournc encore à l'almosphère par évaporation.

La couverture de feuilles mortes est une véritable éponge qui peut retenir par hygroscopéité une tránche pluviale de 7't millimètres avant de rien laisser écouler par ruissellement. On peut dire que le ruissellement est à peu près complètement supprimé sur les versants couverts de forêts en bon étal; où la couverture est respectée.

En forêt, la neige s'amoncelle mieux, fond plus tardivement et plus lentement en imprégnant le sol. Une fois la couche humique saluréc, une portion de liquide pénètre dans les profondeurs du terrain et va, soit alimenter les sources, soit humecter les racines des arbres qui la renvoient dans l'air par respiration. D'après M. Von HöHNEL, naturaliste allemand, un hectare de forêt de roo ans peut exiger journellement de 25 à 30 tomnes d'eau, ce qui correspond à une hauteur de $2 \mathrm{~mm}$. 5 à 3 millimètres de pluie.

Il n'y aura done que le surplus de la pluie qui n'aura pas été évaporé ou absorbé qui pourra ruisseler sur la pente, el ces filets liquides superficiels, arrêlés à chaque pas par le réseau des radicelles, ne gagnent que lentement les thalwegs sans pouvoir engendrer de crues dangereuses. Cependant il faut se garder d'attribuer à la forêt un pouvoir de rétention indéfini : sa puissance absorbante a une limile el lorsque le fcuillage, ala couverture morte, le sol, sont salurés d'humidité, il faut bien que l'eau en excès s'écoule ; mais à ce moment encore, la forêt, si clle n'agit plus guère au point de vie hydrologique, n'en joue pas moins un rôle de première importance pour le maintien des terres sur les pentes. A la suite de grandes pluies prolongées, il y aura inondation, mais pas de déchirures en monlagne et parlant pas de laves torrentielles.

Au point de vue climatérique, la forêt exerce également une influence non moins nette, dans les régions boisćes; en effet, $\mathbf{r}^{\circ}$ les maxima de température sont très alténués ct les minima moins prononcés; la résultante est un abaissement de température moyenne annuelle; $2^{\circ}$ la pluviosité augmente.

L'importance de ces actions diverses dépend de la nalure des essences, el des méthodes culturales appliquées.

Comme on peut traiter les massifs boisés de diverses manières, en taillis simples ou composés, en futaic régulièrc ou jardinée, les exploiter à blanc éloc, en jardinant, en furetant, en éclaircissant, il convient de rechercher quelle sorle de forêt'convient le mieux.

Le taillis se coupe d'ordinaire par grandes surfaces el à intervalles très rapprochés : 30 ans, 35 ans au plus. Trop souvent découvert, le sol reste exposé aux chocs des pluies et des grêles; l'humus s'oxyde rapidement el ne jouc plus, pendant un certain nombre d'années, le ròle d'éponge qui prévenait le ruissellement et partant les érosions. Pour les fulaies, la coupe blanche présente les mêmes inconvénients, et si elle a lieu en montagne, elle risque encore d'engendrer de dangereuses avalanches.

Sans présenter de ces périodes criliques, les taillis furelés n'ont ni un couvert assez épais ni un humus assez abondant pour exercer une aclion protectrice lrès éncrgique. D'aillẹrs ils ne dépassent guère l'altilude de 1500 mètres en Savoic et il ne saurail en être question dans les régions plus élevées.

Dans les fulaies résineuses, le mode de laitement peut varier énormément; le plus rudimentaire est la coupe blanche. Mais, alois que le feuillu donne d'abondants rejets, la souche du résineux demeure stérile el on ne doil attendre la régénération que de l'ensemencement naturel ou artificiel ou de la plantation. De même que dans le taillis, après l'abatage, le sol est vite dépouillé de sa couverture morle et il dencure, chose grave, exposé beaucoup plus longlemps aux actions météorologiques. Le peuplement est, en effet, beaucoup plus lent à se reconstituer, même si l'on plante, ef a fortiori si on attend la germination des graines que le vent apportera peu à peu, que dans le taillis où il n'est pás rare de voir des rejets vigourcux former un épais fourré en 4 ou 5 ans. Pendant loute cette période, le versant reste exposé aux érosions, aux ravinements, aux glissements de neige. Il faut done que le massif soit toujours bien complet. C'est un résultat qu'on obtient en traitant la futaic, soit par la méthode du réensemencement naturel ct des éclair- 
cies (qui a cependant l'inconvénient en montagne, où les vents sont souvent très violents, de donner de nombreux chablis), soịt par la méthode du jardinage, c'est-à-dire en culevant çà et là un ou plusieur's arbres.

La fulaie, et surtoul la futaie résineuse, jouit au plus haut degré des diverses propriétés de fixer le terrain et d'empêcher le ruissellement par ses racines, de le protéger contre les actions météorologiques, d'absorber par sa couverture morte el ses racines une quantité d'eau considérable. Le commentaire qui précède permet donc de préciser ce qu'il faut entendre par forêt dans les formules de Surell.

Dans le chapitre suivant M. Mougin décrit la nature géologique du sol de la Savoie. Il étudie successivement la chaîne du Jura, les Préalpes, les chaînes subalpines comprenant les massifs du Platé, des Bornes, des Bauges et de la Chartreuse, puis enfin les Alpes et les terrains éruptifs. Dans chaque chaîne et chaque massif l'auteur décrit méthodiquement l'ère secondairé, l'ère tertiaire et l'ère quaternaire en y étudiant les systèmes jurassique, crétacé et liasique et triasique.

Ce chapitre se termine par les "Terrains torrentiels" que nous citons en entier.

\section{LES TERRAINS TORRENTIELS}

D'après l'exposé qui précède, on peut indiquer quels élages géologiques sonl le. plus favorables au développement de l'action torrentielle en Savoie.

Les roches éruptives, granits; protogynes, etc..., d'une fạcon générale, sont peu exposées au ravinement, non plus que les gneiss et terrains primitifs. Mais dans les schistes cristallins, on commence à trouver quelques couches plus tendres. Certains schistes à séricites se désagrègent assez aisément et il pourra y naître des ravins (la Corbière à SaintAlban-d'Hurtières, le Nant Cortet à Cohennoz).

Dans les formations permo-carbonifères, les schistes et grès houillers donnant, par leur décomposition, des sols liès meubles et très perméables, perdant leur cohésioñ par suite d'un excès d'humidité, arrivent à engendrer, sous l'action érosive des eaux, de très dangereux torrents. (Le Pousset à Orelle, la Grollaz à Beaune).

Mais ce sont surtout les gypses tendres, solubles, du trias qui sont le plus exposés aux affouillements. Sous l'action des eaux souterraines, il se produit des galeries, des cavernes qui, tôt ou tard, s'effondrent; il peut arriver qu'aucune fracture ne se manjfeste à la superficie, mais généralement ces tassements se produisent à la surface par des entonnoirs très caractéristiques. Un ruisseau s'engouffre-t-il dans ces dépressions, il reparaît rarement et on ne peut prévoir ni l'ampleur, ni l'endroit des débâcles qui s'y produiront tôt ou tard (Arbonne à Bourg-Saint-Maurice ; Merderel de Fontcouverte).

Pour n'être pas cachée comme dans les gypses, l'action des eaux n'est pas moins redoutable lorsqu'elle s'exerce sur les schistes et les marnes noirs du lias. Mềme sur des pentes très faibles, ces terres argileuses, sous l'influence d'une humidité persistante, se mettent à fluer cn coulées pâteuses : il suffit donc d'un ruisselet pour y creuser un sillon profond qui va sans cesse s'agrandissant aux dépens des rives. Mille filets d'eau, dont l'originé est le plus souvent un léger pli du sol ou une déchirure de la berge, gagnent de plus en plus au loin, tentacules qui rongent la montagne et en apportent les débris à la pieuvre géante qu'est le torrent principal. Toutes ces particules terreuses glissent en masses boueuses, envahissent la vallée, recouvrent les cultures, me- nacent les voies de communication et les villages (Rieu Bel à Villargondran, Bon Rieu de Jarrier).

Dans le système jurassique, le dogger (bathonien et bajocien) avec ses couches marneuses est également très affouillable. Aussi y rencontre-t-on des torrents et des ravins (lo Nant Trouble à Ugine, le Brevon à Vailly).

Les schistes et calcaires marneux du berriasien et du valanginien (syslème crétacé), très tendres, sont aussi fréquemment décapés et érodés (torrent de Reninges à Passy, torrent de Mafond à Sixt).

De l'ère tertiaire, on ne trouve guère que des grès, des conglomérats et des schistes ardoisiers, en général, assez résistants. Aussi n'y voit-on guère de déchirures torrentielles. Cependant on peul citer le grand glissement de Mont-Denis dans un sol produit par la désagrégation de ces couches et le ravin du Bouchet où menacent de se produire des glissements de fond.

$\mathrm{Au}$ fond des vallées, il n'est pas rare de voir les rivièrcs remanier les alluvions anciennes ou récentes, ronger le pied de terrasses glaciaires qui se décapent peu à peu et s'éboulent dans le thalweg, faute de cohésion entre lcs divers éléments dont elles sont composées.

Mais à côté de la nature même du sol, il est encore un autre élément qui favorise ou contrarie l'action torrentielle, c'est la disposition des couches. Il est clair que si les roches présentent une texture imbriquée, les eaux, les neiges glisseront à leur surface sans y causer grand dégât. Mais si les diverses assises se présentent normalement à la pente, l'humidité pénètre mieux dans leurs interstices, la gelée les disloque et, au dégel, des masses de matériaux vont s'effondrer dans le thalweg voisin. Survienne un orage ou une brusque fonte de neige, et voilà tous ces débris entraînés d'un seul coup jusque dans la plaine (La Gruvaz à Cevins).

Enfin, il est certains cours d'eau qui ne peuvent qu'avoir un caractère torrentiel : ce sont céux qui sortent des glaciers. Les glaciers charrient et déposent à leurs fronts une quantité de boues et de pierres qu'ils ont reçues dans leur parcours, par suite de la désagrégation des roches supérieures, ou que, par suite des frictions énergiques exercées, ils ont arrachées aux parois encaissantes. Chaque crue aura don pour conséquence la reprise et le remaniement de ces moraines (torrent des Pellerin à Chamonix) et il est clair que ce processus continuera tant qu'il y aura des glaciers.

\section{II. .. LE CLIMAT DE LA SAVOIE CONSIDÉRATIONS GÉNÉRALES}

La Savoie se trouvant sous le $\iota_{4} 6^{\circ}$ de latitude Nord devrait jouir d'un climat tempéré; mais la présence de massifs montagneux puissants atteignant l'altitude la plus grande de toute l'Europe (4 8ro mètres au Mont-Blanc), à côté do vallées basses (2 I2 mètres sur le Rhône) font que l'on y rencontre les climats les plus divers.

A elle seule, la succession des végétaux suffirait à indiquer aux esprits les moins observateurs les différences climatériques des diverses régions savoisiennes. Alors qué dans la Combe de Saroie, on rencontre le figuier, l'aman: dier et autres fruitiers, plus en amont, le noyer, le châtaignier, la vigne subsistent. Ces essences disparaissent ainsi quc le chênc et sont remplacées par les résineux, le pin sylvestre et le sapin d'abord, le mélèze, l'épicéa, le pin cembro ensuite. L'arolle finit même par devenir rare et par céder la place aux pelouses au-dessus desquelles règne l'éternelle stérilité.

C'est devenu une banalité que de dire que la température s'abaisse au fur et à mesure qu'augmente l'altitude et quêt, 
plus on se trouve hảut au-dessus du niveau de la mer, plus les précipitations affectent la forme solide. En continuant a s'élever, on trouve une zone où les condensations ne sont plus que ncige, grêle ou grésil, une région où lc sol demeure constamment couvert de frimas. On sait maintenant que los massifs montagneux favorisent les précipitations, principalement lorsqu'ils sont boisés et, par conséquent, on peut prévoir qu'en Savoie le régime pluvial pourra différer suivant les vallées, d'après la hauteur et le taux de boisement des versants qui les constituent comme aussi suivant les saisons. L'importance des pluies est: un facteur très important de l'action torrentielle. Il est d'observation, en effet, que les crues brutales n'ont lieu qu'à la suite d'une brusque fonte des neiges ou d'un violent orage, ou encore à la suite d'une pluie très prolongée.

La distribution des précipitations n'a pas moins d'intérêt au point de vue végétal : suivant le cas; le terrain sera desséché la plupart du temps et ensuite inondé, sans être imbibé par de courtes et soudaines averses, et il ne nourrira que des plantes xérophiles, ou bien, mouillé régulièrement, il conservera toujours le taux d'humidité indispensable à la vie du plus grand nombre des espèces herbacées ou ligneuses. Les condensations elles-mêmes dépendent du régime des vents dans la région considérée et, en montagne, il n'est pas rare de voir des courants aériens spéciaux dans chaque valléc. Une longue suite d'observations poursuivies ì Annecy depuis ${ }_{773}$ par le Docteur Despine et le chanoine Vaullet ; à Chambéry, depuis I 786, par Dacquin, par le chanoine (depuis Cardinal) Br.uet et par les professeurs du grand Séminaire et enfin, après l'annexion, les bulletins ct carnets des commissions de météorologie et les observations faites par l'administration des Ponts et Chaussées fournissent unc quantité de documents précieux pour l'histoire météorologique de la Savoie. Combien peu de départements pourraient en offrir autant?

\section{LES VENTS}

La Savoie, au point de vue des vents, subit l'influence de l'Océan Atlantique et les dépressions constatées à l'Ouest de la France y arrivent environ de 24 à 36 heures après avoir été observées à Paris.

Mais, par sa situation au milieu du bassin du Rhône, la Savoie est encore soumise aux perturbations atmosphériques de la Méditerranée. Ce sont les vents chauds et humides du Sud, qui souvent, en hiver ou au printemps, amènent la fusion brusque des neiges et des inondations soudaines. Au contraire, les vents $d u$ N., du N.-F. et du N.-W. sont froids et secs.

Venant du golfe de Gascogne, les vents de. S.-W. sont chauds, chargés de vapeur d'eau, amènent la pluie et fréquemment, en été, des orages. Plusieurs de ces vents ont reçu des noms spéciaux dans la région. Ainsi le vent du Nord s'appelle bise ; la bise noire est un vent d'entre Nord et NordEst, très froid, qui chasse les nuages le long des crêtes et finit par les dissiper : on aurait remarqué que la bise noire souflle périodiquement pendant trois ou neuf jours.

On désigne simplement, sous le nom de vent, le vent du Sud : le vent d'Ouest est connu à Chambéry sous le nom de Traverse, parce qu'il franchit la chaîne de l'Epine, et celui d'Est sous le nom de Matinière, parce qu'il souffle surtout au lever du soleil. Le vent d'Est est froid, un peu hu. mide, car il passe par dessus les Alpes toujours plus ou moins enneigées : aussi, en hiver, amène-t-il fréquemment la neige. A côté de ces grands courants d'air qui s'étendent sur toute la Savoie, il y a dans le fond de certaines vallées des vents spéciaux qui n'ont lieu qu'en été.

Le régime des vents n'est pas demeuré constant et ce sont tantôl les vents d'entre N.-E. et N.-W., tantòt les vents d'W. ct S.-W qui dominent.

Sont qualifiés vents du Nord les vents soufflant du N.-N.-E. au N.-N.-W.; vents d'Ouesl, ceux soufflant du N.-W. à l'W.; vents du S.-W., ceux venant d'entre le W.-S.-W. et le S.-S.-W. ; vents du Sud, ceux soufflant du S. au S.-E., el vents d'Est, ceux soufflant de l'E.-S.-E. au N.-E.

Aussi bien à Annecy qu'à Chambéry, depuis $r S_{90}$, ce sont les vents chauds et humides d'entre N.-W. et S.-E. qui dominent de beaucoup : ils soufflent ici pendant 8 á et là pendant 85 pour roo des jours de l'année, soit les $5 / 6$ du temps. On peut donc a priori affirmer que les précipitations seront plus fréquentes avec ces vents.

La comparaison des tableaux des vents à Annecy et à Chambéry fait ressortir la plus grande fréquence des vents du Nord à Annecy, des vents d'Est à Chambéry. Bien que situées dans des vallées largement ouvertes, ces villes se trouvent néanmoins à proximilé de massifs montagneux importants qui modifient profondément la direction générale des courants d'air.

Mais dans l'ensemble, Chambéry et Annecy ont bien le même régime des vents.

Si l'on s'enfonce dans la région alpine. on constate au contraire que les vallées souvent étroites, resserrées, dominées par des cimes très élevées, sont parcourues par des vents qui semblent n'avoir aucun rapport avec ceux qui soufflent de l'Océan ou de la Méditerranée. Des arbres penchés du côté d'amont montrent par leur inclinaison la constance et la force de ces courants : ce phénomène est général et peut s'obscrver dans les vallées de l'Isère, de l'Arve ct de l'Arc.

Ces brises ont des caractères spéciaux, faciles à distinguer : elles n'existent pas durant la mauvaise saison; mais c'est de mai à septembre qu'elles se font sentir, lorsqu'il fait beau, car elles cessent par la pluic ou les temps couverts. C'est vers 8 ou 9 heures du matin que ces vents se lèvent, remontent les vallées, croissent en intensité jusque vers 4 heures, puis diminuent peu à peu vers 7 ou 8 heures du soir ; ce sont des vents inférieurs, qu'on ne trouve plus à quelques centaines de mètres au-dessus du thalweg.

Après un moment de calme, vers 9 heures du soir, il se lève un autre vent moins violent qui descend la vallée jus; qu'à l'apparition du soleil.

Ces brises périodiques ont été étudiées en Maurienne par le Cardinal Billiet, à Moutiers par M. Roche, Directeur des Royales-Salines. Dans cette localité, la brise diurne remonte le cours de l'Isère et vient du défilé d'Aigueblanche. La nocturne arive par les vallées du Doron de Bozel et de l'Isère dú côté d'Aime.

En Tarentaise, comme en Maurienne, il souffle aussi même dans la journée, lors des mauvais temps, un vent, d'unc violence extrême qui, après avoir franchi la chaîne frontière, descend la vallée. Ce vent d'Italie s'appelle, dans la région de Bourg-Saint-Maurice, vent du Petit Saint-Bernard, et dans celle de Lans-le-Bourg, la Lombarde. Quand se lève la Lombarde, on voit apparaître sur la cime des montagnes les brouillards, nés sur Je versant de la Doire, qui se déversent sur les pentes occidentales des Alpes en nappes continues et amènent la pluie, à moins qu'un autre courant d'air, franchissant le massif de la Vanoise et, pour ce motif, est désigné sous ce nom; ne vienne refouler, après les avoir 
mises en lambeaux, les brumes du Piémont, après une lutte souvent prolongée, mais toujours tumultueusc.

L'intensité des vents, les mouvements tourbillonnaires de l'atmosphère qui, ainsi que l'a démontré M. J. Vallot pour le massif du Mont-Blanc, vont en croissanl avec. l'altitude, sont, nolamment vers les crêtes, un obstacle au développement de la végétation forestière et l'origine d'avalanches. A co point de vuc encore, l'influence des vents sur le régime des eaux est loin d'être négligeable. Mais nous ne possédons aucune mensuration sur la puissance et la persistance des violents courants aériens dans les hautes régions.

\section{LA TEMPÉRATURE}

Un des plus importants facteurs du climat, au point de vuc spécial qui nous occupe et dont la connaissance est du phus haut intérêl, est la température. Pour qu'ils puissent ćvolucr, grandir, fruclifier, les végétaux ont besoin d'une certaine somme de chaleur et dans une station donnée on pourra conclure, toutes choses égales d'ailleurs, que telle espèce ligneusc ne saurait vivre si elle n'y trouve la quantité de calories qui lui est nécessaire annuellement.

On connaît quelques remarques anciennes sur la douceur de certains hivers, ce qui permit aux fleurs de remplacer dans les prés la neige qui normalement eût dû les couvrir, aux moissons de mûrir très tôt. Mais on ne saurait tirer de conclusions de ces faits isolés, anormaux, au sujet du climat de la région savoisienne.

Jleureusement on possède une longue série d'observations thermigues faites à Annecy, depuis $177^{3}$ jusqu'à nos jours, par le docteur Despine, lc chanoine Vaullet et la Commission de Météorologic.

Elles montrent un accroissement continuel de la température moyenne depuis $177^{3}$ jusqu'en $x 859$ : de 'l'annexion de la Savoic à la France jusqu'à l'époque actuelle, cette température semble s'être légèrement abaisséc. Les dates délimitant les diverses périodes de temps n'ont pas élé choisies au hasard : $179^{2}$ a vu la région savoisienne se donner à la Riépublique française el marque donc la fin de l'ancien régime en même temps ıque l'origine de grands déboisements renouvelés, mais pendant moins longtemps, à la chute de f'Empire; $181 / 4-1860$ le début et la fin de la période Sarde.

Au point de vuc torrentiel, il y a à fairc des rapprochements entre ces variations de la température moyenne annuclle et l'étendue forestière durant les diverses périodes considérées.

Sauf pour les deux villes d'Anccy et de Chambéry, on ne trouve guère de documents relatifs à la température. Dans la Haute-Savoie, il existe actucllement dix stations où se font. journellement des observations thermiques.

II serait intéressant de connaître les températures dans chacune des grandes vallées de Savoie, en divers points; mais à part la vallée de l'Arve qui possède trois stations, Annemasse, Bonneville et Chamonix, ailleurs on est réduit à calculcr la température moyenne d'après l'altitude du lieu au-dessus d'une localitẹ où la température moyenne annuelle est connue, en diminuant de $x^{\circ}$ cette température par $180 \mathrm{~m}$. de différence de niveau. Comme on ne tient compte ni des courants d'air dominants, ni du relicf, de la configuration et de li nalure du sol, ni de l'exposition, il est évident qu'on n'oblient par celte méthode qu'une approximation très insuffisante pour déterminer si une plante peut recevoir la quantité de chaleur qui est indispensable à son existence en un endroit donné. "C'cst d'ailleurs ce qu'a fait ressortir
M. J. VatLot (Annales de l'Observatoire Météorologique du Mont-Blanc, tome I, p. $3 \mathrm{I}$ ).

\section{LES PRÉCIPITATIONS ATMOSPHÉRIQUES}

Pour évoluer, les plantes n'ont pas seulement besoin de chalcur, il leur faut aussi une certaine quantité d'eau : il est donc nécessaire de connaître Je régime pluvial de la région car, suivant que l'humidité sera abondante ou non, la flore pourra présenter des caractères fort différents.

La manière dont se répartit la lame d'eau qui tombe annuellement n'est pas non plus sans influence sur la végétation et surtout sur le régime des cours d'eau. Si les pluies se produisent d'une manière uniforme pendant toute l'année, il est certain que le débit des rivières et ruisseaux no variera que dans de faibles proportions et il ne saurait y amener des érosions bien importantes : il n'en est pas de même, si les précipitations sont rares, mais extrêmement brusques et abondantes. Par conséquent, à côté de la lame d'eau qui tombe annuellement, il faut tenir compte du nombre de jours pendant lesquels se font les condensations atmosphériques.

Malheureusement les observations faites à Annecy par le docteur Despine ct le chanoine Vaullet n'ont pas porté sur la lame d'eau annuelle.

A Chambéry, Dacquin, médecin en chef des hôpitaux, fait des mensurations pluviométriques de 1786 à $\mathbf{1} 8 \mathbf{1} 2$ environ, mais l'on ne connaît que celles de ${ }_{7} 86$ à 1802 .

D'autres observations ont été exécutées au Grand Séminaire à Chambéry, au milieu du $\mathrm{xIx}^{0}$ siècle; à partir de I839 le pluviomètre élait placé dans le jardin de cet établissement à $\mathrm{I}^{\mathrm{m}} 3 \mathrm{o}$ au-dessus du sol; mais on n'en trouve que ce qui a été communiqué aux journaux de l'époque, c'està-dire assez peu.

Ce n'est que depuis 1862 à Chambéry et, depuis 1870 , ’̀ Annecy, que des précipitations ont été notées d'une façon suivie : les résullats constatés ont été publiés dans les bulletins des commissions météorologiques des deux départements savoisiens el dans le Bulletin Central Météorologique en France.

Diverses stations ont été créées, munies de pluviomètres et dont les observations consignées dans les bulletins météorologiques annuels permettent de se faire une idée de la répartition des pluies dans les diverses vallées.

Enfin le service du reboisement de la $5^{\mathrm{e}}$ Conservation a organisé diverses expériences pour déterminer l'importance des précipitations suivant l'altitude en un certain nombre de points élagés sur le même flanc d'une montagne.

\section{Des Variations des Précipitations annuelles à Chambéry et Annecy}

Comme pour les températures, il convient de rechercher d'abord les variations qu'a pu subir la pluviosité dans les mêmes stations depuis les premières observations faites ef d'en faire ressortir le caractère.

On ne connaît pas, sauf pour les années 1839 -1840-184iI8:33, la valeur des précipitations constatées au pluviomètre du Grand Séminaire de Chambéry. Mais dans la Revue Savoisienne (r858, tome III, p. 38), on lit que la lame d'ealu moyenne annuelle observée à Chambéry est de $\mathrm{o}^{\mathrm{m}} 93 \mathrm{o}$. Co chiffre serait la moyenne soit de la période $183_{9}-1857$, soit de la dernière décennie $1848-185_{7}$. Il est d'ailleurs corroboré par la valeur de la lame d'cau moyenne annuelle donnéé par les premières observations faites par le service dé 
Ponts et Chaussées, de r863 à ı870, qui est en effet égale à 959 millimètres.

A Annecy, on voit que la moyenne annuelle du nombre des jours de pluie ou de neige qui était, avant l'annexion de $179^{2}$, de 134 , tombe à I 25 pendant la Révolution et l'Empire ; elle se relève sous la Restauration Sarde pour diminuer ensuite. Le chiffre de 137 qui s'applique à l'intervalle 18 15-1824 n'est qu'un accident venant de ce que la période envisagéc est trop courte, ro ans. Si on joint à la période suivante, de manière à comprendre les années de I8r5 à 1859 , on reste au-dessous du chiffre moyen de 133 jours pluvieux par an.

Dans l'ensemble, il y a donc bien une diminution du nombre des jours de pluie ou de neige à Annecy.

A Chambéry, si on n'a pas de tels renseignements, on possède cependant des données partielles sur la lame d'eau annuelle.

D'après le docteur Dacquin, de $\mathrm{r}_{7} 86$ à $\mathrm{r} 802$, il serait tombé cn moyenne à Chambéry $\mathrm{I}^{\mathrm{m}} \mathrm{I} 22$ d'eau; il faut observer que cette moyenne comprend dix années de l'époque révolutionnaire et six ans seulement de l'ancien régime.

Au milicu du $\operatorname{xix}^{\mathrm{e}}$ siècle, la précipitation moyenne annulle n'est plus que de 930 millimètres.

Mais à partir de l'annexion à la France, l'importance des condensations augmente.

Si on rapproche de ces observations la diminution de l'étendue et de la densité des massifs boisés pendant la: même temps, on verra aisément que les variations de la température et des pluies sont intimément liées à la déforestation du sol de la Savoie et, depuis r 860 , à la conservation plus efficace de ce qui restait des grandes étenducs sylvestres d'autrefois et qu'elles n'en sont que les consćquences naturelles.

\section{Importance des précipitations atmosphériques annuelles en différentes stations de Savoie}

Ainsi que nous l'avons dit plus haut, les mensurations pluviométriques les plus anciennes, en dehors de celles faites à Chambéry et à Annecy, ne remontent guère au delà de 35 ans.

Les tableaux qui résument d'un coup d'œil les observalions faites dans les divers bassins des plus importantes rivières de la Savoie conduisent à faire les remarques suivantes :

Remarque I. - Bien qu'on ne puisse comparer mathématiquement les quantités d'ea'u tombées dans les différentes stations et qui sont les moyennes fournies par des séries d'années très différentes, dont l'origine n'est pas identique, les résullats donnés permettent néanmoins de se faire une idée de la répartition des pluies dans toute l'étendue de la Savoie. D'une façon générale, l'importance de la lame d'eau annuelle diminue à mesure que l'on descend vers le Sud.

Les vallées de Dranses, de l'Arve, du Fier, de la Leysse et la Combe-de-Savoie reçoivent des précipitations abondantes qui arrivent ordinairement à dépasser I mètre par an.

Dans le bassin de la Haute-Isère, les bessins secondaires de l'Arly et du Doron de Beaufort participent à ce même régime, tandis que la vallée de Tarentaise se fait remarquer par un climat plus sec.

La Maurienne offre le même caractèrc, mais beaucoup plus accentué et il faut noter que les deux seules stations où la lame d'eau annuelle dépasse I mètre sont : $\mathrm{r}^{\circ}$ le MontCenis à plus de 2 ooo mètres d'altitude $; 2^{\circ}$ le fort de Montgilbert sis sur la crête qui sépare la Moyenne-Isère de la
Basse-Maurienne et qui, par suite, pourrait êlre compris dans la Combe-de-Savoie.

Remarque 11 . - Il convient aussi d'observer que la zone de grande pluviosilé n'englobe que les préalpes et les chaînes subalpines. Au fur et à mesure que l'on s'enfonce dans les Alpes, on voit diminuer l'importance des précipitations. Les observations faites dans le département de la Savoie ne datent, en effet, que de $\mathrm{x} 890$ et il faut considérer la même période dans la Haute-Savoie.

Ainsi, dans la partie de la vallée de l'Arve comprise dans le massif alpin, à Chamonix, la lame d'eau moyenne annuclle est moindre que dans le reste du Faucigny.

Quand on pénètre en Tarentaise, on voit également les précipitations perdre de leur fréquence et de leur abondance ; elles présentent, à Moutiers, un minimum bien accusé, puis elles décroissent jusqu'à Val-d'Isère à partir de Bourg-Saint-Maurice, où la situation de cette localité au débouché des vallées de Tignes, du Reclus et des Chapicux favorise très probablement les condensations.

En Maurienne, le nombre des jours pluvieux ou ncigeux, aussi bien que l'épaisseur de la lame d'eau annuclle, décroîl depuis Chamousset jusqu'à Modane qui semble bien être le point le plus sec des deux départements. Cette diversité de climats allant depuis le régime brumeux et humide du Nord jusqu'à la sécheresse lumineuse des Haules-Alpes démontre surabondamment que la Savoie est bien le trait d'union cntre le Nord et le Midi, entre la Bourgogne et la FranchcComté d'une part et la Provence de l'autre.

Remarque III. - Les notations pluviométriques failes dans les vallées de l'Arc et de l'Isère sont trop peu anciennes i 890 ) pour qu'il soit possible d'affirmer que ce phénomènc de réduction des précipitations atmosphériques, au fur et à mesure que l'on remonte le cours des rivières alpines, a loujours existé.

Il semble, à considérer ce qui s'est passé sur l'Arve, que celte inversion soit de date récente. Jusqu'à Sallanche, c'està-dire dans toute la zone subalpine, la pluviosité crôit avec l'altitude, que l'on considère la période r89 I-I905 ou la période antérieure.

A Chamonix, au contraire, pour la période $\mathrm{x} 89 \mathrm{x}-\mathrm{y} 905$, la lame d'eau moyenne annuelle n'est que de $785^{\mathrm{mm}} \mathrm{o6}$, infórieure à celles constatées dans le même temps à Annemasse $\left(\tau 95^{\mathrm{mm}} \mathrm{o} 2\right)$, à Bonneville $\left(94 \mathrm{x}^{\mathrm{mm}} \mathrm{o} 6\right)$, à Sallanche ( $\left.1 \mathrm{ro} 3^{\mathrm{mm}} \mathrm{o} 6\right)$. Pour la période antérieure $1876-1890$, on a observé à Chamonix une précipitation moyenne annuelle de ${ }_{1} 38^{\mathrm{mm}} \mathrm{O} 2$, súpérieure à toutes celles observées dans les autres stations.

De ce fait, il semble que l'on doive en rapprocher un autre : $189_{2}$ a marqué le maximum de la crue glaciaire do la fin du $x_{I x}{ }^{e}$ siècle dans le massif du Mont-Blanc. A ce moment, en effet, le front du glacier des Bossons n'était plus qu'à 3 I 8 mètres de la croix plantée en i 8 I 8 à l'exlrémité de la moraine, alors qu'aujourd'hui la langue terminale de ce glacier est à 300 mètres plus amont. Depuis quinze ans, les glaciers ont fondu énormément, des surfaces de roche et de terre considérables ont apparu dépouillées de neige, réduisant ainsi la superficie glacée qui favorise certainement les condensations. Cetle fusion in tense s'est fait également sentir sur les glaciers de la HauteTarentaise et de la Haute-Maurienne et certains petits glaciers suspendus ont même complètement disparu. Les plus grands des glaciers ont rétrogradé et se sont affaissés, par exemple le glacier des sources de l'Arc et celui de la Galise, 
pour ne prendre que ceux qui engendrent deux des plus imporlanles rivières de la Savoie.

La diminution de la pluviosité est-elle la conséquence de la régression des apparcils glaciaires ou celle-ci résultc-t-elle de la rarelé et de la pauvrelé des précipilations?

Avant 1890 , la température moyenne annuelle était, à Ghamonix, de $+6^{\circ} 39$ (période $1880-1887$ ) ; après la période 1 899 -1 905 , elle n'est plus que de $+4^{\circ} 29$. Par conséquent, l'intensité de la fusion des glaciers du massif du Mont-Blanc élail plus considérable avant 1890 .

les éléments météorologiques, actuellement connus, ne pernetlent guère de résoudre d'une façon certaine ce problème de climatologie des régions alpines qui mérite de lixer l'attention.

Remarque IV. - Il n'était pas moins intéressant de vérifier si les précipitations atmosphériques qui, d'une façon générale, d'après les tableaux, semblent croître avec l'allilude, vont en augmentant sur un même versant de monlagne, à mesure qu'on s'élève, d'une manière continue ou si, au contraire, clles passent par un maximum.

l'Administration des Eaux et Forêts a fait établir entre les: Houches (Haute-Savoie) et le glacier de Tête-Rousse, au Sul-Ouest du massif du Mont-Blanc, six tubes Vallot de ? mèlres de hauteur et dont l'ouverture a $0^{\mathrm{m}} \mathrm{o} 8$ de diamètre. Comme ces récepleurs, par'la forme de leurs dimensions, ne permeltent qu'à une partie seulement des pluies ou des neiges de parvenir jusqu'à la cuvetle où on les recucille, on ne saurait comparer les résultats obtenus avec les données du pluviomètre.

Mais comme les récepteurs sont tous identiques, les indicalions qu'il fournissent sont comparables entre elles.

II cxiste un maximum très accusé au plateau des Rognes c'est d'ailleurs le point où flottent le plus souvent les nuages et les brumes et on pouvait prévoir que les régions situées à un niveau supérieur à 2550 mètres recoivent moins de pluic et de neige que celles placées au-dessous.

Une autre série de récepteurs a également été installée en Maurienne entre Modane-Fourneaux $\left(10 / 0^{m}\right)$ et le sommel de la Belle-Plinier à 3 og m mètres; on constale également une augmentalion des précipitations avec l'altitude. mais le maximum n'est pas très accusé.

\section{Intensité des Précipitations journalières}

Il ne suffit pas dans une élude torrenticlle de connaîle la valcur de la précipitation annuelle et le nombre des journées pluvieuses, il faut aussi savoir comment se réparlissent les pluies; il est clair, en effet, que s'il tombe $20 \mathrm{~mm}$. d'eau en ro minutes, on observera des érosions el des phénomènes éncrgiques de décapage qui ne se produiront pas si la chute se produit en 24 heures.

Malheureusement les bullelins météorologiques nc renscignent pas sur la durée des averses, les observateurs n'allant relever leur pluviomètre qu'à hcure fixe, tous les jours. li n'y a guère que dans les établissements météorologiques disposant d'un personnel spécial que l'on note de tels renseignements ; mais parfois certains observateurs les signalent cependant avec détail.

Les précipitations les plus abondantes ont, en général, licu au moment des orages. C'est ainsi qu'en Maurienne, au poste militaire du Fréjus, à 2550 mètres d'altitude, on a recueilli, le 23 juillet $\mathrm{r} 906,63^{\mathrm{mm}}$ d'eau en 2 heures environ.

De même, à Annecy, le I I juillet Igoh, en 40 minutes, de $8 \mathrm{~h}$. ro à $8 \mathrm{~h}$. 50 du matin; il tombe $22^{\mathrm{mm}} \mathrm{i}$ de pluie, et à Thonon, le $1 \mathrm{I}$ août suivant, $2^{\mathrm{mm}}$ en 15 minutes à partir de 2 heures du soir.

En 19o5, à Annecy, le pluviomètre accuse, le I4 mars, de 9 hcures du matin à midi, $14 \mathrm{~mm}$. ; le 19 mai, de 5 h. $40^{\circ}$ à 5 h. 52 du soir, $3^{\mathrm{mm}} 9$; le 14 juin, de 3 h. 15 à 4 heures du soir, $12^{\mathrm{mm} 5}$; lc $\mathrm{r} 7$ juin, en $\mathrm{I} 5$ minutes, $17 \mathrm{~mm}$. A Chambéry, le 2 I aoûl $\mathrm{I} 888$, ainsi que le 7 septembre suivant, il tombe i $8 \mathrm{~mm}$. en 15 minules; le $3 \mathrm{x}$ mai 1889 , de $4 \mathrm{~h} .25$ à 5 heures du soir, la lame d'eau recueillie atteint une épaisseur de 26 millimètres.

En I 890 , le 25 mai, une pluic orageuse donne, en un quart d'heure, $\mathrm{r}_{2} \mathrm{~mm}$. au pluviomètre; le I2 juin, une ondée de même durée n'a que $6 \mathrm{~mm}$. et une fournit I I $\mathrm{mm}$. d'eau en 25 minutes.

Mais si les plus récents bulletins de la Commission météorologique de la Haule-Savoie donnenl de semblables indicalions, il n'en est pas de même des aulres documents ; aussi faut-il se borner à un état des précipitations supérieures à 30 millimètres en 24 heures.

Des tableaux dressés à ce. sujet il résulle que :

$\mathrm{I}^{\circ}$ Le nombre des journées de grande pluie va, en général, cn augmentant avec l'allitude, suivant une même vallée, ainsi que le nombre annuel des jours de pluie (Dranses, Arve, Fier, Leysse, Arc) ;

$2^{\circ} \mathrm{L}^{\prime}$ augmentation des jours de grande pluie ne varie pas comme celle des jours pluvieux de l'année, elle croît plus vile, comme le fait ressortir le pourcentage calculé d'après les données des tableaux ;

$3^{\circ}$ Dans la vallée de la Haute-Isère, le nombre des jours de grande pluie et leur fréquence en regard du nombre annuel des jours de pluie, offre les mêmes maxima ct minima que l'épaisseur de la lame d'eau moyennc annuell en Tarentaise;

$4^{\circ}$ C'est dans le bassin du Fier que les grandes pluies sont le plus fréquentes et dans celui de l'Arc qu'elles sont le plus rares;

$5^{\circ}$ Bien qu'on re puisse comparer en valeur absolue l'imporlance des grandes pluies, à cause des différences de longueurs dans les périodes d'observations, c'est en Maurienne que les maxima des précipitations en 24 heures sont lo moins élevées ; de même, c'est en Maurienne que le nombro moyen annuel des jours de grandes pluies est le moins fort

Ces remarques semblent être en opposition absolue avec ce que l'on sait du caractèrc torrentiel de la vallée de l'Arc. Il suffit de se rappeler, pour faire tomber cette antinomic apparente, que si la torrentialité dépend de la grandeur des pentes et du déboisement elle est aussi la résultante de la violence et de la courte durée des averses. Or, les documents météorologiques ne donnent aucune idée de ces facteurs. Un exemple fora mieux saisir l'importance du temps: le 24 juillet I 906 , il a été recueilli du poste militaire de Fréjus, en deux heures, une lame d'ieau de 63 millimètresi Le bulletin météorologique indiquera seulement ces 63 millimètres pour l'intervalle compris entre 29 juillet à 9 heures du matin et le 25 juillet à pareille heure, soit une moyenú de $5_{2}^{\mathrm{mm}} 25$ pour deux heures au lieu de 63 millimètres. On sait assez ce qu'ont été les conséquences de ce "sac d'eau" de $63 \mathrm{~mm}$., alors qu'une pluie de $5^{\mathrm{mm}} 25$ en deux heures n'aurait produit absolument aucun dégât ;

$6^{\circ}$ Les précipitations atmosphériques supérieures à Ioo millimètres en 24 heures ne sont pas très rares; elles ne calscnt ccpendant de sérieux ravages que dans le cas où elles sont précédées ou suivies d'autres pluies assez abondantes cl où clles déterminent une brusque fusion des neiges tom- 
bées antérieurement (la Dranse, en octobre 1888 ; la Leysse, le 18 janvier 1875 et le 14 janvicr 1899 ).

Ce sont là des cas particuliers qui sont examinés dans l'historique des torrents.

\section{Les chutes de Neiges en Savoie}

La connaissance des chutes de neige est également des plus importantes. A maintes reprises, la brusque fusion de masses neigeuses considérables accumulées sur les montagnes amène des inondations dans les vallées principales ou détermine d'énormes érosions dans le fond des ruisseaux el des ravins.

En se précipitant sur les pentes des montagnes, au printemps, sous forme d'avalanches de fond, les nappes de neige exercent sur le sol des frictions énergiques, brisent ou déracinent les arbres, arrachent des pierres, de la terre. Après leur fusion, les cônes d'avalanche laissent sur place, généralemenl dans des thalwegs, un amas de débris de toute nature, minéraux et végétaux. Survienne une brusque averse ct tous ces débris sont charriés vers la plaine sous forme d'une lave qui souvent ne devra rien à l'affouillement du lit ou des berges du cours d'eau.

D'une façon plus indirecte, la neige contribue à créer ou à aggraver l'irrégularité du régime des cours d'eau alpins. Lors des grands froids de l'hiver, elle s'étend sur les versants déboisés en couches pulvérulentes sans cohésion et dont la pente d'équilibre est des plus réduite.

La chute d'une corniche, un ébranlement de l'air ou du sol, le passage d'un animal suffisent à rompre cet état d'équilibre instable : une avalanche de poussière est née. En glissanl, la poussière glacée éprouve de la part de l'atmosphère une résistance de plus en plus grande, elle s'étale en nuées dont le poids, croissant à chaque instant, refoule puissamment les couches aériennes. Bientôt c'est un ouragan qui se précipite vers les thalweg's, qui en suit les contours et qui, sous son souffle, renverse, casse les arbres situés sur son parcours ou même sur le versant opposé. On conçoit que la destruction instantanée des cantons entiers de forêts contribue éncrgiquement à la torrentialité du cours d'eau. dans le bassin duquel ils se trouvaient.

Enfin, la persistance des neiges, conséquence de leur abondance et de la température locale, peut être un obstacle au développement de certaines espèces végélales. On trouve en liaute montagne une limite variable, suivant les temps et les localités, au-dessus de laquelle les neiges ne disparaissent plus tous les ans.

A.-Abondance et fréquence des précipitations neigeuses. - Toutes choses égales d'ailleurs, il est évident que les précipitations neigeuses croîtront avec l'altitude; mais le régime neigeux n'est pas le même pour toutes les vallées savoisiennes, pas plus d'aillcurs que le régime des pluics.

Depuis l'hįver 1899-ז9oo, l'Administration des Eaux et Forêts a organisé dans les deux départements de la Savoie un service d'observations nivomélriques. Auparavant, on n'avail de renseignements que dans le bulletin météorologique de la Haute-Savoie : encore fallait-il tenir compte de ce fait que les observateurs ne mesuraient l'ẹpaisseur de la couche neigeuse qu'une fois par jour, au moment où ils relevaient le contenu du pluviomètre. De cette pratique il résulte qu'on ne peul adopter comme exacte l'épaisseur de neige donnée, car il a pu se produire des tasscments, des fusions partielles. La densité réclle de la neige ne peut s'obtenir qu'en la notant aussitôt après la chule, avant qu'aucun tassement ail en lieu; elle est d'ailleurs des plus va- riables suivant les circonstances. Ainsi par temps chaud $\left(+3^{\circ}\right)$, à Chambéry, on a trouvé (hiver I 899-19oo) qu'ì I $\mathrm{mm}$. d'eau de fusion correspondaient seulement $2^{\mathrm{mm}}$ I de neige. Par contre, à Bessans, par - $15^{\circ}$, pendant l'hiver rgo4-rgo5, il n'a pas fallu moins de $\mathrm{r}^{3} \mathrm{x}^{\mathrm{mm}} 5$ de neige pour donner la même quantité d'eau de fusion.

Ces différences de densité correspondant à divers états de la neige (collante ou pulvérulente) expliquent très bien les cffets mécaniques variés que causent les avalanches, suivant leur nature.

Dans une même station, la densité moyenne de la neige varie suivant les hivers; ainsi, à Chambéry, pendant l'hiver' r $903-1904$, il a fallu en moyenne $6^{\mathrm{mm}} 2$ de neige pour donner I millimètre d'eau de fusion ; pendant l'hiver rgoo-rgor, il en a fallu i 5 millimètres.

Les observations nivométriques ont lieu chaque année, du $\mathrm{x}^{\text {er }}$ octobre au 3 I mai.

Elles conduisent aux observations suivantes:

Remarque I. - Ainsi qu'on pouvait le prévoir, les précipitations neigeuses augmentent avec l'allitude, en quantité el en fréquence. Mais cet accroissement varic suivant les vallées. C'est la Maurienne qui présente l'accroisscment le plus faible.

Remarque II. - D'une façon générale, la quantité moyenne de neige nécessaire pour donner I millimètre d'eau de fusion va également en augmentant avec l'altitude ; mais elle n'est pas identique pour des points également élevés audessus du niveau de la mer.

Remarque III. - Il convient de ne pas adopler les chiffres du tablcau comme l'expression exacte du régime neigeux de la région savoisienne. La période d'observations porte au maximum sur huit années et il s'est trouvé qu'ì part le dernier les hivers onl ćté extrêmement peu neigeux. Par suite, les résultats obtenus se trouvent inférieurs a la moyenne réelle applicable aux deux départements. Ainsi, d'après le bulletin de la Commission météorologique de la Haute-Savoie dans la période r876-rgor, à Annecy, il y aurait eu en moyenne, par an, $2 x$ jours neigcux ct une lame d'eau de $8 o^{\mathrm{mm}}{ }_{2} \delta$ provenant de la fusion de la neige, alors que les chiffres correspondants tirés du tableau sont respeclivement de 17 jours et $53^{\mathrm{mm}} 3$.

Mais pour pouvoir élablir la répartilion des neiges suivant les localités, il a fallu nécessairement se boner ì prendre la période réduite pendant laquelle des observations ont élé parlout poursuivies d’une façon identique dans toute la Savoic.

B. - Les Avalanches. - Tes avalanches sont des masses neigeuses ou glacées qui se précipilent sur les pentes des montagnes et roulent vers les vallées. D'une façon générale, on peut distinguer trois sortes d'avalanches :

$x^{\circ}$ L'avalanche de fond, qui entraine toule la neige en masse compacte, lourde, tassée, et laisse le sol à nu; on l'appelle aussi quelquefois, pour cela, avalanche terrière. Ordinairement c'est au printemps qu'elle se produit, quand le tassement el une fonte partielle alourdissent la neige et que les surfaces de contact entre la neige et le sol sont fortement réduites ou que le coefficient de frottement à la surface du versant s'est trouvé diminué par le ruissellement des eaux. Les quanlités de neige entrainces sont parfois extrêmement importantes el les cônes qui forment les avalanches au pied de la montagne arrivent à être assez épais pour ne pas disparâtre pendant l'été, malgré l'élévalion de la température ot la faiblesse de l'altitude. Ces avalanches de fond dégradent le sol, soit par friction, soit par choc; elles 
entraînent les terres, les blocs, les arbres qu'elles trouvent sur leur passage. Aussi, généralement, les couloirs que suivent ces avalanches, car la plupart ont des passages fixes, ne présentent-ils que le roc vif ou un tapis herbacé continu ('t sans aspérités ;

$2^{\circ}$. L'avalanche de poussière ne met en mouvement que des neiges très légères, sans cohésion, faites de très petits cristaux glacés, tels qu'il en tombe par les grands froids. Quand la masse neigeuse est assez forte, comme les couches n'ont pour ainsi dire aucune cohésion cntrc elles, elles se mettent à fluer sur la pente. L'air heurté par la vague neigeuse oppose une résistance croissante et bienlôt les paillettes s'élèvent en nuages dont la densité, l'ampleur et la vitesse vont en croissant. De plus en plus comprimé, l'air se précipite, s'engouffre dans les vallées en ouragan, abat les arbres, enlève les toits des chalets, jonche le sol de débris qu'une fine poussière glacée vient bientôt recouvrir d'un blanc linceul. Il n'est pas rare de voir des cantons entiers de forêts, situés sur le versant opposé à celui où s'est
Comme il est intéressant, au point de vue de la fixation des neiges et de la correction des torrents, de savoir si les avalanches prennent naissance au-dessus ou au-dessous do la limite supérieure de la végétation forestière, l'AdminisIration des Eaux et Forêls, depuis rgoo, a fait établir en Savoie une statistique des avalanches.

Mais un tableau récapitulatif ne peut évidemment donner que des résultats incomplets. Certaines avalanches affectent des formes diverses : tantôt elles se produisent comme avalanches de fond, tantôt comme avalanches de poussière, tantôt comme avalanches superficielles de l'un ou l'autre type. (Voir tableau ci-après.)

Les unes ont lieu une fois seulement par an, les autres plusieurs fois, d'autres enfin sont rares. Dans le cours d'un même hiver, le même couloir peut engendrer des avalanches de nature différente.

Les nombres donnés ne comprennent que les avalanches ayant suivi des routes différentes et non pas le total des avalanches tombées pendant la période considérée.

ÉtAT DES AVALANCHES ET DE LEURS DÉgats DU fer JANVIER 1900 AU 31 MaI 1907

\begin{tabular}{|c|c|c|c|c|c|c|c|c|c|c|c|c|c|c|c|c|c|}
\hline & & $\begin{array}{l}\text { OMBRI } \\
\text { AVALAN }\end{array}$ & & & $\begin{array}{r}0 \\
\text { D'AVAL }\end{array}$ & CHES & & $\stackrel{\mathscr{0}}{\Xi}$ & 趈 & $\begin{array}{l}\text { BÉTA } \\
\text { ENSE }\end{array}$ & & DE PER & $\begin{array}{l}\text { TOMBRI } \\
\text { NNES EN }\end{array}$ & VELIES & 总 & \% : & \\
\hline $\begin{array}{c}\text { DES } \\
\text { BASSINS }\end{array}$ & 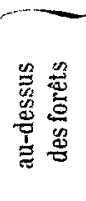 & 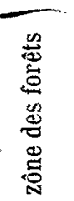 & 恿 & $\begin{array}{l}\bar{\Xi} \\
\Xi \\
\stackrel{\Xi}{0}\end{array}$ & 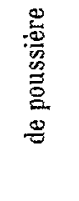 & 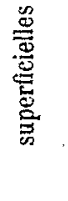 & 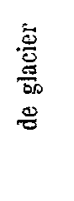 & 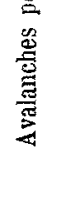 & 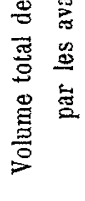 & 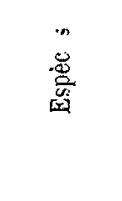 & 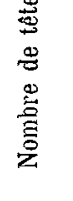 & 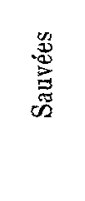 & 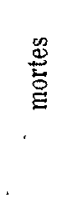 & $\stackrel{\vec{T}}{\vec{E}}$ & 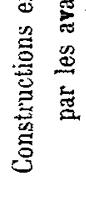 & 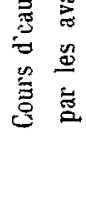 & 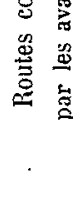 \\
\hline Less Dranses & 1 & 86 & $8 i$ & 86 & 2 & 12 & 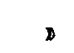 & 68 & 499 & $D$ & » & 2 & 1 & 3 & 7 & 24 & i) \\
\hline Anve & 97 & 83 & 180 & 74 & 4 & 20 & 2 & 122 & 3638 & moutons & 49 & " & D & ) & 7 & 9 & 24 \\
\hline Fien & 7 & 160 & 167 & 81 & 10 & 9 & $n$ & 66 & 783 & $"$ & $"$ & $D$ & 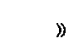 & $n$ & 14 & 20 & 19 \\
\hline Lersse & ") & 29 & 29 & 77 & 6 & 17 & $"$ & 17 & 75 & ") & D & $"$ & $»$ & $D$ & $D$ & $"$ & D \\
\hline HAUTE-IsÊRE & 111 & 216 & 327 & 60 & 4 & 36 & ) & 192 & 1338 & $\begin{array}{l}\text { hovius } \\
\text { moutons }\end{array}$ & $\begin{array}{r}4 \\
68\end{array}$ & 17 & 9 & $: 6$ & 48 & 84 & 180 \\
\hline Anc & 193 & 143 & 336 & 60 & 33 & 67 & 0,3 & 218 & 2688 & moutons & 70 & 66 & 8 & 74 & 14 & 142 & 63 \\
\hline Tolaux moyens & 409 & 717 & 1126 & D & $"$ & 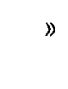 & 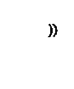 & 683 & 9021 & $\begin{array}{l}\text { buvins } \\
\text { moutons }\end{array}$ & $\begin{array}{r}4 \\
187\end{array}$ & 85 & 18 & 103 & 90 & 279 & 291 \\
\hline 0/0 Moyen génér. & D & x & $D$ & 69,7 & 3,9 & 26 & 0,4 & \# & ) & $D$ & " & » & 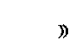 & » & » & » & 》 \\
\hline
\end{tabular}

produite l'avalanche, renversés par la violence du courant d'air produit par ces avalanches ol Jes liges de sapins, des épicéas ou des mélèzes sont couchées en éventail.

On peut concevoir des modalités de ces deux lypes d'avalanche. Ainsi, par exemple, une couche de neige déjà ancienne a subi une fusion partielle el s'est congelée superficiellement. Une nouvelle précipitation neigcuse se produit. Il arrive que cette neige fraîche, déposée sur un plan incliné très glissant, manquant d'adhérence se mette en mouvement en donnant naissance, suivant les conditions de tempéralure, soit à une avalanche de fond, soil à une avalanche de poussière, mais qui, cette fois, sera superficielle ;

$3^{\circ}$ Ce sont des décollements du front des glaciers qui engendrent les avalanches de glacier. Ces éboulements peuvent parfois porter sur des quantités énormes de glace et, s'ils dominent des rocs escarpés, ils peuvent déterminer de véritables catastrophes, soil en écrasant des chalets, des villages, soit en amenant des débâcles qui submergent toute une vallée.
Comme avalanches périodiques, il n'a été compté que celles qui descendent par les mêmes couloirs, à des intervalles plus ou moins rapprochés, une ou plusieur's fois par an, ou tous les deux ans ou plus rarement.

Le pourcentage des avalanches de toutes natures a été pris sur le chiffre total des avalanches observées pendant la période $\mathrm{I}^{\text {er }}$ janvier $\mathbf{I} 900-3$ I mai $\mathbf{1 9 0 7}$.

On voit, d'après le tableau qui précède, combien d'arbres peuvent détruire les avalanches.

Ges phénomènes sont aussi des agents de décapage éner-

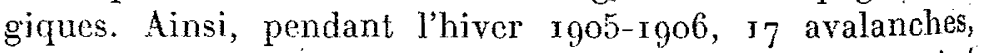
sur $x 3$ communes du bassin de la Haute-Isèrc, ont entraîné 329 mètres cubes de terre et 12 mètres cubes de blocs; dans ro communes de Maurienne, 64 avalanches ont transporté 2576 mètres cubes de terre et 22 blocs cubant en tout 12 mètres cubes 200 ; dans 4 communes seulement du bassin de l'Arve, 35 avalanches ont emmené 177.2 mètres cubés de matériaux de tous genres, dont un bloc de 8 mètres cubes.

Si l'on remarque que ces constatations ont été faites dans 
des régions facilement accessibles et que, dans certains vallons des montagnes, à grande altitude, où la désagrégation est très active, mais totalement déserts pendant 9 à ro mois de l'année, il se produit des avalanches énormes que l'on n'observe pas, ayant, par suite, une puissance d'érosion considérable, on peut conclure que les avalanches fournissent aux cours d'eau alpestres une quantité de matériaux de charriage qui n'est pas négligeable.

Cela n'a rien qui doive étonner, car certains cônes d'avalanche atteignent de fortes dimensions. Ainsi, à Beaufort, l'avalanche de la Gevrotière a couvert 60 ares et atteignail 10 ono mètres cubes ; à Cevins, l'avalanche du pont du Dard s'étalait sur 90 ares et avait 24 ooo mètres cubes de neige lassée; à Saint-Julien-de-Maurienne, le 9 avril ıgo4, une énorme avalanche s'abattait dans la combe des Essarts sur I hectare 92 et avait une épaisscur de 15 mètres; 12 jours après, dans la même commune, I hectare $72 \mathrm{du}$ ravin de l'Aiguille disparaissait sous une couche de neige de 8 à ro mètres. Le 20 mars I9o7, à Chamonix, l'avalanche des Chosalets occupait une surface de 2 hectares $7^{\circ}$ avec près de roo ooo mètres cubes de neige ; ̀̀ Sixt, à la même époque, l'avalanche des Follys avait 5 hectares de superficie et un volume de 66 ooo mètres cubes!

\section{(à suivre).}

\section{PROCÉDÉ POUR L'AMÉLIORATION DU FACTEUR DE PUISSANGE}

Causes p'un mauvais faGteur de puissance dans les insTALLATIONS Triphasées. - Le mauvais facleur de puissance que l'on conslate souvent dans les installations triphasces lient en général au branchement au réseau de moteurs d'induction et principalement de gros moteurs à marche lente. Mais les moteurs à grande vitesse peuvent exercer également une influence défavorable s'ils tournent à faible charge ou à vide. Pour mettre ce point en évidence, rappelons d'abord en quoi consiste le facteur de puissance :

Tout moteur a besoin d'excitation ; mais tandis que pour les moteurs synchrones (comme pour les moteurs à courant continu) l'excitation est fournie par une source de courant continu, les moteurs asynchrones doivent emprunter au réseau le courant nécessaire à leur excitation ; ils prennent donc au réseau deux sortes de courant : un courant qui sert à la production du travail et l'autre qui sert à l'excitation ou à la magnétisation. La représentation graphique de ces

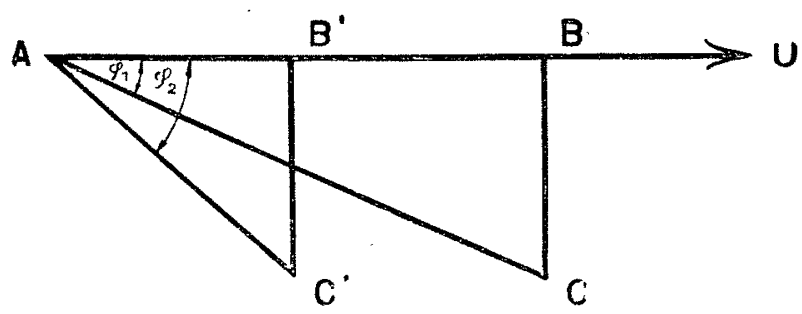

FIG I.

courants est donnée par la figure $\mathrm{I}$; $\mathrm{AB}$ est le courant de travail, BG le courant de magnétisation. Celui-ci qui ne produit pas de puissance et est appelé en conséquence déwatté, est en arrière de $90^{\circ}$ par rapport à la tension du réseau, c'est-à-dire que dans le diagramme vectoriel, il est perpendiculaire au vecteur du courant de travail qui est un courant watlé. $\mathrm{AC}$, résultante des deux courants composants, représente le courant total emprunté au réseau.
L'angle BAC mesure le décalage de phase entre la tension et le courant; son cosinus est désigné sous le nom de facteur de puissance. Le courant d'excitation a une valeur à peu près indépendante de la charge de sorte qu'unc diminution de celle-ci, c'est-à-dire du courant de travail, fait croître l'angle de décalage ; à vide, la composante wattée atteint son minimum ; le facteur de puissance descend à o, ro-or5 et le moteur prend au réseau environ $40 \% \mathrm{du}$ courant de pleine charge. Moins un moteur d'induction est chargé, plus est faible son facteur de puissance, comme il ressort clairement de la figure I. En outre cette figure I montre que le facteur de puissance du moteur d'induction ne peut jamais atteindre l'unité par suite de la présence du courant d'excitation.

Des essais rigoureux ont montré que les installations industrielles qui sont équipées avec des moteurs triphasés ont en général un facteur de puissance compris entre 0,6 et o,85; la limile supérieure n'est atteinte que rarement.

Inconvénients d'un mauvais facteur de puissance. - Le conrant d'excitation nécessaire aux moteurs asynchrones doit être fourni en même temps que le courant de travail par les génératrices de la centrale. L'échauffement admissible pour ces dernières limite le courant qu'on peut leur faire débiter quelle qu'en soit d'ailleurs la répartilion en watlé et déwatté ; les génératrices ne seront complètemenl utilisćes que pour $\cos \varphi=\mathbf{r}$. Si les moteurs asynchrones connectés au réseau, par suite de leur mauvais facteur de puissance prennent aux génératrices une forte proportion de courant magnétisant, il ne restera que peu de courant disponible pour le travail. Par exemple un facteur de puissance égal à 0,5 ne permettra d'utiliser que la moilić du courant total pour produire du travail.

Ce raisonnement s'applique également aux transformateurs et aux lignes de distribution dont l'utilisation en ce cas ne sera que partielle.

Il faut encore tenir compte de ce que les pertes ducs au courant déwatté, assez réduites pour les génératrices et les transformateurs, peuvent, pour les lignes de distribution, atteindre dans certains cas une grande valeur. Ces pertes croissent comme le carré de l'intensité du courant de sorte que si l'énergie électrique doit être transportée à grande distance, l'amélioration du facteur de puissance réduira les pertes d'énergic d'unc façon très sensible. Il est donc de grande importance de trouver un moyen approprié d'améliorer le facteur de puissance, c'est-à-dire de compenser lc décalage de phase.

De plus, lorsque les réseaux s'étendent, il arrive que, par suite de l'accroissement du débit des lignes, la chite de tension augmente de telle sorte que le réglage de l'excitation des générateurs ne suffit plus à tenir la tension normale du réseau. Dans ce cas on peut, par un léger déphasage en avant, réduire la chute de tension à une valeur acceptable.

S'il s'agit de créer une nouvelle installation de puissance donnée, l'amélioration du facteur de puissance correspond à une diminution des frais d'installation et d'exploitation.

Pour une centrale existante qui travaille avec un mauvais facteur de puissance, l'amélioration de celui-ci donne également des avantages sensibles, spécialement si la centrale est déjà arrivéc aux limites de sa capacité. Par des moyens relativement peu coûteux, l'amélioration du facteur de puissance permet d'augmenter la puissance de la centrale sans installer de nouvelles unités; le matériel, machines et canalisations est mieux utilisé et le rendement de l'ensemble se trouve accru. 